\title{
FROM TAKING TO MAKING PARADOX: A MULTI-LEVEL PERSPECTIVE ON HOW CEOS BALANCE NESTED PARADOXES
}

\author{
MICHAEL SMETS, \\ Saïd Business School, University of Oxford, Park End Street, OX1 1HP, UK; \\ michael.smets@sbs.ox.ac.uk \\ AMANDA MOSS-COWAN \\ College of Business Administration, University of Rhode Island \\ ANDROMACHI ATHANASOPOULOU \\ Queen Mary Univ. of London \& Saïd Business School \\ CHRIS MOOS \\ Saïd Business School, University of Oxford \\ TIM MORRIS \\ Saïd Business School, University of Oxford
}

\section{INTRODUCTION}

As environments have come to be increasingly complex and volatile, many organizations have become "many things to so many different people" (Kerr, 1963: 8). As a result, they often face related but contradictory pressures that persist over time, such as the paradoxical tension between the need to explore and exploit simultaneously (Andriopoulos \& Lewis, 2009). CEOs, as organizational leaders, who are operating at the intersection of their organization and its institutional environment are uniquely exposed to those tensions. However, while there is extensive knowledge about how CEOs experience tensions at institutional and organizational levels, little is known about where CEOs experience what kind of tensions, i.e. across individual, organizational, or institutional levels. Further, given the assumption that CEOs are passive 'takers', rather than active 'makers' of the tensions they encounter (Raisch, Hargrave and Van de Ven, 2018), little is known about why those tensions matter to CEOs in the first place and how CEOs, in their daily practice as leaders, deal with paradoxical tensions. To address this, we develop a multi-level perspective on the paradoxical tensions CEOs experience at the individual level, as an organizational leader, and a corporate diplomat interfacing with the organization's external environment. This multi-level understanding explores what paradoxical tensions they host - personally and on behalf of their organization, where those tensions are located, why CEOs constitute some paradoxes as salient, and how they sustain these in their everyday practice.

The prior literature on paradox has provided important insights into how leaders can deal with institutional logics that are interrelated, yet persistently opposed to each other (Lewis \& Smith, 2011), for example through integration and balance of a duality of demands (Baxter \& Montgomery, 1996), or reframing and transcending the tensions between them (Poole \& Van de Ven, 1989). Yet, little is known about why paradox matters to organizational leaders in the first place, that is, what creates paradox salience for them (Schad \& Bansal, 2018). A prevailing assumption is that paradoxical tensions simply exist in the organizational environment and become salient in situations of scarcity, change and innovation, and plurality and competition 
(Schad, Lewis, Raisch \& Smith, 2016; Smith \& Lewis, 2011). This suggests CEOs operate with little agency in relation to the tensions that they face. Second, there is a large practitioner literature, and an emerging scholarly understanding of how leaders practically deal with opposing yet persistently interrelated demands in their daily lives (e.g. Bolden, Witzel \& Linacre, 2016). For instance, scholars have identified 'paradox-savvy' leader behaviors (Waldman \& Bowen, 2016) and the benefits of a 'paradox mindset' (Miron-Spektor, Ingram, Keller, Smith \& Lewis, 2018). Yet, there is still limited systematic knowledge about how CEOs, in their daily leadership practice, deal with paradoxical tensions. Third, the literature on paradox has largely assumed that paradoxes appear either at the level of the institutional field (e.g. Bartunek \& Rynes, 2014; Chung \& Beamish, 2010), the organization (e.g. Andriopoulos \& Lewis, 2009; Raisch \& Birkinshaw, 2008; Smith \& Tushman, 2005), or to a lesser extent, the individual leader (e.g. Miron-Spektor et al., 2018; Smith, 2014; Waldman \& Bowen, 2016; Zhang et al., 2015). Few studies have considered that paradoxes can surface and manifest at multiple levels (e.g. Andriopoulos \& Lewis, 2009; Bradach, 1997; Schreyogg \& Sydow, 2010). This focus on separate levels of analysis limits the ability to comprehend how leaders deal with paradoxes, given their frequently 'nested' characteristics (Jarzabkowski, Le \& Van de Ven, 2013; Lewis, 2000). Looking across multiple levels enables us to provide a more realistic, and dynamic model of how leaders deal with tensions.

To gain a more realistic and dynamic understanding of the why, what, where and how CEOs deal with tensions, we initiated a research project that examines the question, "How do CEOs develop the competence to lead in a changing world?" To answer it, we conducted a large scale qualitative study involving rich and personal conversations with 146 global CEOs from a wide range of sectors and cultures, providing us with unique insights into their world- their leadership, challenges and decision making process. Being elite managers and hence notoriously difficult to gain access to (Kakabadse et al., 2015), these CEOs constitute a research sample particularly unique and highly insightful. Such elites can provide a 'big picture' perspective that most research usually cannot provide (Marshall \& Rossman, 1999).

\section{FINDINGS}

Many of the CEOs we interviewed felt caught "at the intersection" of environmental and organizational demands. One observed: "once business was impervious. You could operate and the world around you could be a vortex of activity, but you could just keep going. Now, with the combination of all the factors at play, business and what's happening outside are actually one" [003]. Both between and within levels, these demands are often contradictory: CEOs must take decisions to move their organization forward yet face scrutiny from both internal and external stakeholders. CEOs report that, as more stakeholders make competing-yet equally validdemands, they face paradoxical choices "between right and right", rather than just right and wrong. It is such dilemmas that make decisions so vexing and alignment so difficult. Many paradoxes do not queue up neatly to be dealt with in sequence, but trouble CEOs simultaneously.

Based on our inductive analysis, we identified what paradoxical tensions CEOs host, where they locate those paradoxes (i.e. at the leader/individual, organizational or institutional level), how CEOs practically manage those paradoxes on a daily basis, and why CEOs constitute some paradoxes as salient and not others. To illustrate these relationships, we use the metaphor of a chemical reaction. In a chemical reaction, a catalyst, i.e. "a substance that increases the rate of a chemical reaction" (Oxford English Dictionary, 2019a), triggers a chemical reaction 
between a 'stimulant', i.e. "something that increases activity, interest, or enthusiasm in a specified field" (Oxford English Dictionary, 2019b), and a 'reactant', i.e. "a substance that takes part in and undergoes change during a reaction" (Oxford English Dictionary, 2019c). The CEO acts as a catalyst in the active construction of the tension (the why) between a 'paradox stimulant' and a 'paradox reactant' (the what). The 'paradox stimulant' is the first part of the tension, which the catalyst, i.e. the CEO, connects to the 'paradox reactant', which is the second part of the tension. Like in chemistry, the catalyst is not a necessary condition for the functioning of the reaction, which can also be triggered by environmental conditions (Lewis \& Smith, 2011). For example, for employees within the organization, the paradoxical tension between work and life can be salient without the intervention of the CEO as the catalyst. However, through their actions, and their command over organizational culture, structure and resources, CEOs can make that tension more or less salient. The CEO can thus act alone, or in interaction with environmental conditions, in actively constructing the salience of paradoxes.

Our framework shows that the 'paradox stimulant' and 'reactant' can be situated at either the same, or different levels of analysis (the where), thereby creating vertical connections between paradoxical tensions on the institutional, individual, and organizational levels. For instance, one CEO \{the catalyst\} stated that "the question as to why a CEO's tenure is shorter now, in part it's because of restless institutional investors \{the stimulant, i.e. the institutional environment $\}$ and Boards always think the grass is greener, but it's also that these jobs kind of kill really you or burn out people \{the reactant, i.e. the individual leader\}. [...] That's part of the advantage of that bifocal approach, the ability to focus on the short-term \{first part of the paradox and the longer \{second part of the paradox\}" [005].

Alternatively, some tensions can be constituted on the same level. For instance, one CEO \{the catalyst\} stated that “there's a company's need to be responsible ffirst part of the paradox to a number of stakeholders, including the shareholders [...] We have a lot of shareholders who are very focused on [...] improving returns \{second part of the paradox and not so interested in the long term [the stimulant, i.e. the institutional environment $\}.[\ldots]$ But it is a bit of a leap of faith to be honest, there's no guarantees that doing what's right and taking decisions that are in the interest of the long term, [...] will also produce good results the reactant, i.e. the institutional environment $\}$ in the short term. Getting that balance right is certainly not getting any easier" [009].

In previous sections, we outline how CEOs have developed specific practices (the how) of dealing with paradox. Complementing this, our framework demonstrates the relationship between the why, what, and where of paradoxes.

\section{CONTRIBUTIONS}

In showing how, why, and where CEOs deal with what kind of tensions, we make three contributions to the paradox literatures. First, in showing that CEOs can constitute tensions as salient, we provide an important counterpoint to the assumption that paradoxical tensions simply exist in the organizational environment and become salient through environmental conditions over which individual actors have little influence (Schad et al., 2016; Smith \& Lewis, 2011). We show that CEOs have a much more agentic role in 'making' rather than just 'taking' paradox, providing empirical evidence for the agentic construction of the salience of tensions (Schad \& Bansal, 2018). 
Second, we extend the idea of "nested paradoxes" (Lewis, 2000) to develop an integrated multi-level understanding of how CEOs deal with tensions. Existing scholarship has focused on how leaders can make "paradoxical enquiries" with the help of consultants or action researchers (Lüscher \& Lewis, 2008: 235), cognitively develop elaborate "paradox mindsets" (MironSpektor et al., 2018), engage in dynamic decision making to address paradoxes (Smith, 2014), and deploy complex paradoxical leader behaviors (Zhang et al., 2015). In contrast, to the current literature, which has emphasised their "timeless, abstract" (Poole \& Van de Ven, 1989: 565) nature, we show how leaders normalise paradox through their everyday work practices, rather than high-level and abstract mindsets. Indeed, CEOs develop very practical approaches of managing paradox, some of which are paradoxical in their own right. We therefore situate paradox management within the everyday practice of organizational leadership.

Third, through our dynamic multi-level framework, we highlight what kind of paradoxes CEOs deal with and where different tensions are located at the institutional, organizational, and individual levels. In so doing, we extend Smith and Lewis' (2011) notion of holding competing demands in a "dynamic equilibrium" across levels. While the previous literature has assumed that paradoxes operate at the same individual, organizational, or institutional level (Schad et al., 2016), we show that paradox management can take on a vertical dimension with the two opposing 'poles' of paradoxes located at different levels. We, therefore, reach beyond simplified notions of dyadic contradictions that have dominated the paradox, ambidexterity and institutional complexity literatures to paint a more realistic picture of $21^{\text {st }}$ century leadership challenges.

REFERENCES AVAILABLE FROM THE AUTHORS 
Copyright of Academy of Management Annual Meeting Proceedings is the property of Academy of Management and its content may not be copied or emailed to multiple sites or posted to a listserv without the copyright holder's express written permission. However, users may print, download, or email articles for individual use. 